\title{
Klippel - Feil Syndrome Associated with Atrial Septal Defect
}

\author{
Ramush Bejiqi ${ }^{1}$, Ragip Retkoceri ${ }^{1}$, Hana Bejiqi ${ }^{2}$, Naim Zeka ${ }^{1}$, Arlinda Maloku ${ }^{1}$, Majlinda Berisha ${ }^{1}$ \\ Division of Cardiology, Pediatric Clinic, University Clinical Center of Kosovo, Prishtina, Republic of Kosovo ${ }^{1}$ \\ Main Center of Family Medicine, Prishtina, Republic of Kosovo²
}

\begin{abstract}
ntroduction: Three major features result from this abnormality: a short neck, a limited range of motion in the neck, and a low hairline at the back of the head. Most affected people have one or two of these characteristic features. Less than half of all individuals with Klippel-Feil syndrome have all three classic features of this condition. The etiology of Klippel-Feil syndrome and its associated conditions is unknown. The syndrome can present with a variety of other clinical syndromes, including fetal alcohol syndrome, Goldenhar syndrome, anomalies of the extremities etc. Associated anomalies occur in the auditory system, neural axis, cardiovascular system, and the musculoskeletal system. Results and discussion: Cardiovascular anomalies, mainly septal defects, were found in 7 patients in Hensinger's series, with 4 of these individuals requiring corrective surgery. In our case we have had registered a nonrestrictive atrial septal defect and corrective surgical intervention at age 18 months in the Santa Rosa Children's Hospital (USA) has been done successfully. Careful examinations of specialist exclude anomalies in other organs and systems. Radiographs and MRI of the thoracic and lumbosacral spine are obtained and other anomalies have been excluded. Key words: Klippel -Feil syndrome, atrial septal defect, echocardiography.
\end{abstract}

Corresponding author: Ramush Bejiqi, MD. Division of Cardiology, Pediatric Clinic, University Clinical Center of Kosovo, Prishtina, Republic of Kosovo. Tel: +37744120129. E-mail: rbejiqi@hotmail.com

\section{INTRODUCTION}

First time described in 1912, from Maurice Klippel and Andre Feil independently, Klippel-Feil syndrome (synonyms: cervical vertebra fusion syndrome, Klippel-Feil deformity, KlippelFeil sequence disorder) is a bone disorder characterized by the abnormal joining (fusion) of two or more spinal bones in the neck (cervical vertebrae), which is present from birth. Three major features result from this abnormality: a short neck, a limited range of motion in the neck, and a low hairline at the back of the head. Most affected people have one or two of these characteristic features. Less than half of all individuals with Klippel-Feil syndrome have all three classic features of this condition (1).
Since first classification from Feil in three categories (I - III) other classification systems have been advocated to describe the anomalies, predict the potential problems, and guide treatment decisions. Patients with Klippel-Feil syndrome usually present with the disease during childhood, but may present later in life $(2,3)$. The challenge to the clinician is to recognize the associated anomalies that can occur with KlippelFeil syndrome and to perform the appropriate workup for diagnosis (4).

\section{CASE REPORT}

A 28-month-old girl, from normal pregnancy and absolutely health parents, weighing $16.3 \mathrm{~kg}$, during the routine pediatric examination systolic murmur was noted and for cardiolog- ical examination at tertiary level was referred. The child's growth and development was completely normal. There was no sweating or fatigue during feeding or normal activities. Complete clinical and cardiological examination was obtained. An arterial blood gas was within normal limits. Clinical examination demonstrated: a quite precordium, normal first heart sound, short midsystolic murmur 2-3/6 degree on the apex and left sternal border, and single second heart sound. Electrocardiogram showed: normal sinus rhythm, left axis deviation, and incomplete bundle branch block. A chest radiogram revealed a normal cardiac silhouette. The pulmonary vasculature was normal in appearance, and there were no infiltrates seen. Cross-sectional echocardiography demonstrated: normal systemic and pulmonary vein connection, big hole, diameter $12 \mathrm{~mm}$, in the middle part of interatrial septum with nonrestrictive left to right flow. There was normal atrioventricular and ventriculo-arterial connection. By continuous Doppler waves and color Doppler trivial tricuspid regurgitation and hyperdynamic flow through the pulmonary artery was noted.

Anteroposterior (AP) as well as lateral neutral, flexion, extension radiographs of the cervical spine demonstrate abnormal development of the upper cervical spine described as follows: There is at least partial fusion of $\mathrm{C} 2$ and $\mathrm{C} 3$, the ossified portion of the dens is 
noted to be inferiorly displaced relative to the anterior arch of $\mathrm{C} 1$, the posterior elements of C1, C2 and likely C3 are hypoplastic/absent, and there is a defect in the lamina of the superior - most vertebral body which contains formed posterior elements. The fused vertebral bodies are small and also have an abnormal anterior convex configuration. On the flexion and extension radiographs, and atlantoaxial instability is identified. The skull projects over most of the cervical spine on the AP views so evaluation for vertebral bodies is limited. The posterior skull is noted to have a "beaten copper” appearance. A cervical rib is present on the right. The paravertebral soft tissues have normal appearance. The nasopharynx, oropharynx, and laryngopharynx are patent and clear. Family history was normal for congenital heart disease, clefts, MR/DD, seizures, hearing loss, eye, Dx, diabetes, other birth defects, and pregnancy losses. Consanguinity is denied.

The etiology of Klippel-Feil syndrome and its associated conditions is unknown. The syndrome can present with a variety of other clinical syndromes, including fetal alcohol syndrome, Goldenhar syndrome, anomalies of the extremities etc. (5). A close evaluation of the immediate family is indicated, because autosomal dominant inheritance with variable expression in affected individuals has been noted, although this is presumably rare. There is a strong association with congenital abnormalities of the genitourinary tract (30 - $40 \%)$, including double collecting systems, renal aplasia and horseshoe kidney (6). Klippel-Feil syndrome is estimated to occur in 1 in 40,000 to 42,000 newborns worldwide. Females seem to be affected slightly more often than males. Gunderson suggested that it is a genetic condition, while Gray found a low incidence of inheritance or (7-12) some type of global fetal insult, which could explain the other associated conditions. Mutations in the GDF6 and GDF3 genes can cause Klippel-Feil syndrome. These genes provide instructions for making proteins that belong to the bone morphogenetic protein family, which is involved in regulating the growth and maturation (differentiation) of bone and cartilage. The protein is involved in setting up boundaries between bones during skeletal development. GDF6 and GDF3 gene mutations that cause Klippel-Feil syndrome likely lead to a reduction in functional protein. Some people with Klippel-Feil syndrome do not have identified mutations in the GDF6 or GDF3 genes. The cause of the condition in these individuals is unknown. When KlippelFeil syndrome is caused by mutations in the GDF6 or GDF3 genes, it is inherited in an autosomal dominant pattern, which means one copy of the altered gene in each cell is sufficient to cause the disorder $(5,6,7,8)$. Sometimes this condition is inherited in an autosomal recessive pattern, which means both copies of a gene in each cell have mutations. However, in these cases, the gene involved is unknown. The parents of an individual with an autosomal recessive condition each carry one copy of the mutated gene, but they typically do not show signs and symptoms of the condition $(9,10)$.

\section{DISCUSSION}

Clinical presentation is varied because of the different associated syndromes and anomalies that can occur in patients with Klippel-Feil syndrome. A complete history and careful physical examination may reveal some associated anomalies. Klippel-Feil syndrome involves the congenital fusion (failure of segmentation) of one or more cervical motion segments, and most patients have associated congenital anomalies of the cervical spine or other organs and systems. These anomalies may occur at the craniocervical junction (occipit-C2), the subaxial spine (below $\mathrm{C} 2$ ), or both (11). In our case have multiple developmental anomalies of the upper cervical spine consistent with KlippelFeil syndrome. Te posterior skull has a "beaten copper" appearance which may represent a normal variant or less likely reflective of gyral impression from increased intracranial pressure because it is only seen posteriorly. Associated anomalies occur in the auditory system, neural axis, cardiovascular system, and the musculoskeletal system. Cardiovascular anomalies, mainly septal defects, were found in 7 patients in Hensinger's series, with 4 of these in- dividuals requiring corrective surgery (12). In our case we have had registered a nonrestrictive atrial septal defect and corrective surgical intervention at age 18 months in the Santa Rosa Children's Hospital (USA) has been done successfully. Careful examinations of specialist exclude anomalies in other organs and systems. Radiographs and MRI of the thoracic and lumbosacral spine are obtained and other anomalies have been excluded. People with Klippel-Feil syndrome may have other features in addition to their spine abnormalities. Some people with this condition have hearing difficulties, genitourinary abnormalities such as malformed kidneys, a type of birth defect that occurs during the development of the brain and spinal cord (neural tube defect), an opening in the roof of the mouth (cleft palate), or heart abnormalities. Affected individuals may have underdeveloped shoulder blades that sit abnormally high on the back, a condition called Sprengel deformity $(13,14)$. Lateral flexionextension radiographs of the cervical spine should be performed on all patients to determine the motion of each open interspace. Clinically, flexion-extension is often maintained if a single functioning open interspace is maintained. Those with hyper mobility of the upper cervical segment are at risk of developing neurologic impairment. Affected individuals with hyper mobility of the lower cervical segment are at increased risk for degenerative disk diseases and should be treated symptomatically $(15,16)$.

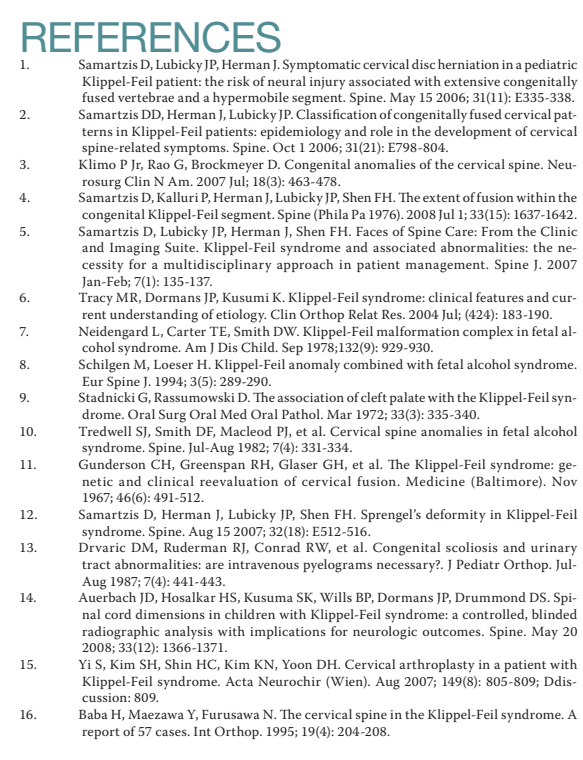

